\title{
IDENTIFICAÇÃO DE ADULTERAÇÃO EM ÓLEOS DE OLIVA: PROBLEMATIZANDO A INTRODUÇÃO À ESPECTROMETRIA DE MASSAS
}

\author{
Angélica Priscila Parussolo Tonin ${ }^{a}$, Gabrielly Ribeiro Carneirob ${ }^{b}$ Marcos Alessandro dos Santos Ribeiro ${ }^{a}$, Jaime da Costa \\ Cedran $^{\mathrm{c}}$, Valquíria de Moraes Silva ${ }^{\mathrm{b}}$ e Eduardo Cesar Meurer ${ }^{\mathrm{b}, *}$ \\ aniversidade Estadual de Maringá, 87020-900 Maringá - PR, Brasil \\ bUniversidade Federal do Paraná, 86900-000 Jandaia do Sul - PR, Brasil \\ 'Universidade Tecnológica Federal do Paraná, Campus Medianeira, 85884-000 Medianeira - PR, Brasil
}

Recebido em 17/04/2018; aceito em 14/05/2018; publicado na web em 28/06/2018

\begin{abstract}
IDENTIFICATION OF ADULTERATION IN OLIVE OIL: PROBLEMATIZING THE INTRODUCTION TO MASS SPECTROMETRY. This work propose the development of an experiment that may be used for the teaching of chemistry for undergraduate students in general, using the analysis of olive oil and soybean oil toward the direct injection electrospray ionization Mass Spectrometry - DI-MS/MS with 18-crown-6 as organic modifier. Firstly, olive oil was analyzed from two different brands, one of reputed brand and the other of popular brand and lower price. Soybean oil of popular brand was also analyzed in order to search possible similarities in the components of these oils and to search adulterations of olive oil with soybean oil. The mass spectra were obtained in positive ion mode of analysis, and allowed us to observe characteristic ions of soybean oil, olive oil, and identify the adulterated olive oil. This simple experiment of easy development may be applied in any institution which has a mass spectrometer with electrospray ionization. In addition, with the use of the experiment, the teacher can illustrate general chemistry concepts such as: molecular mass, preparation of solutions, oxidation, and acid-base reactions.
\end{abstract}

Keywords: mass spectrometry; general chemistry; analysis of oils; adulteration in oils.

\section{INTRODUÇÃO}

A espectrometria de massas, Mass spectrometry (MS), é uma técnica analítica que converte moléculas ou átomos em íons carregados, medindo suas massas e detectando suas abundâncias. Pode também, se o equipamento tiver a capacidade, fragmentar as moléculas para deduzir suas estruturas e, adicionalmente, fornecer informações quantitativas de um analito através de curvas de calibração. Com esta técnica é possível estudar a química dos íons como a entalpia de uma reação, energia de ligações, afinidade entre íons e moléculas neutras em conjunto com cálculos de orbitais moleculares. ${ }^{1}$ Por ser uma técnica que utiliza vários conceitos básicos em química, a aplicação da espectrometria de massas no ensino tem aumentado cada vez mais, já que muitas universidades possuem este tipo de equipamento. Existe uma variedade muito grande de fontes de ionização e analisadores de massas que um equipamento de espectrometria de massas pode ter. Um dos mais simples e robustos é o espectrômetro de massas quadrupolar com ionização por eletrospray, do inglês Eletrospray Ionization (ESI), o que facilita a possibilidade de seu uso em ensino. Um exemplo desta utilização é apresentado no trabalho de Kim, Eckhert e Faull, ${ }^{2}$ que usou a ESI-MS para detectar o complexo do NADH - ácido bórico. Através de um experimento, no qual os alunos de graduação calcularam aritmeticamente a massa do íon negativo mais provável proveniente do $\mathrm{NADH}$, a massa do complexo formado entre NADH e ácido bórico e os espectros adquiridos os permitiu responder a questões sobre a estrutura em fase gasosa desses íons. Em outro estudo, o objetivo foi introduzir a espectrometria de massas com ionização e dessorção a laser assistida por matriz, do inglês Matrix-assisted laser desorption/ionization (MALDI-MS) e a ESI-MS na identificação de ácidos graxos, fosfolipídios e seus produtos de oxidação como técnica analítica na graduação, no qual os alunos de química aprenderam a usar essas técnicas para identificação qualitativa de espécies e medidas quantitativas usando padrões internos. ${ }^{3}$

*e-mail: eduardo.meurer@gmail.com
Segundo Beglinger, ${ }^{4}$ a espectrometria de massas é uma ferramenta que todo acadêmico deveria ter acesso. É uma técnica simples e muito poderosa, devido às suas aplicações em várias áreas, desde monitorar os produtos de uma reação na química orgânica, até a identificação de proteínas. Devido a isso, a introdução da espectrometria de massas como parte da ementa para estudantes na graduação deve se tornar prioridade em toda instituição ensino que possua este equipamento. ${ }^{4}$

Devido às inúmeras aplicações, essa técnica atingiu uma variedade de áreas nunca alcançada por alguma outra técnica analítica, e uma dessas áreas é a da pesquisa em alimentos, a qual tem crescido significativamente nos últimos anos. A análise de pesticidas em tomates feita por Golge e Kabak ${ }^{5}$ utilizou a cromatografia a líquido acoplada à espectrometria de massas sequencial, do inglês high performance liquid chromatography tandem mass spectrometry (HPLC-MS/MS), para a identificação de 109 resíduos de pesticidas. No estudo feito por Afify et al., ${ }^{6}$ que utilizou a mesma técnica citada anteriormente, identificaram-se 150 resíduos de pesticidas em uva. A técnica de espectrometria de massas pode também ser utilizada para análise de óleos vegetais, como por exemplo a técnica de ionização por spray sônico a pressão ambiente, do inglês Easy Ambient Sonic-Spray Ionization (EASI), que foi aplicada com sucesso para avaliar a composição de triacilgliceróis (TAGs), ácidos graxos livres e hidroperóxidos dos óleos. ${ }^{7}$

Este amplo leque de aplicações direciona a $M S$ como técnica de fundamental importância na formação de químicos, engenheiros químicos, engenheiros de alimentos e outros profissionais que utilizam a química como ferramenta. Entretanto, não se deve introduzir a técnica em cursos de graduação somente apresentando os aparelhos e seus constituintes, mas sim propiciar ao acadêmico a oportunidade de vivenciar as diversas aplicações da técnica. Dentre os cursos em que se aborda a espectrometria de massas está a Licenciatura em Química, embora obviamente nas escolas de educação básica esse instrumento não estará presente, o conhecimento da técnica se faz para consolidar conceitos da formação específica do profissional licenciado. Além 
disso, com o aumento exponencial do acesso à informação por parte dos alunos e também pelo crescente interesse em programas de TV, que de alguma forma abordam conceitos científicos (sejam policiais, forenses e etc), a compreensão dessa técnica pode auxiliar o futuro professor a discutir temas relacionados ao assunto com seus alunos.

$\mathrm{Na}$ formação de licenciados, as disciplinas específicas de ensino de química discutem, dentre outros aspectos, a importância do tipo de abordagem da experimentação para a aprendizagem dos alunos. Segundo Ferreira de Sá, ${ }^{8}$ a eficiência no aprendizado de um conteúdo por um estudante está intimamente ligada à forma como o aluno pensa e analisa uma determinada problemática, e isso é específico para cada área de ensino. Neste trabalho se propõe um experimento com características investigativas, pois se acredita que este tipo de atividade apresenta algumas vantagens em relação à abordagem comumente empregada. Nesse sentido, Gil Perez e colaboradores, citados por Suart e Marcondes, ${ }^{9}$ afirmam que:

“muitas das atividades experimentais propostas no ensino têm a concepção empírico-endutivista, a qual evidencia o papel da observação e da experimentação "neutra", esquecendo o papel essencial das hipóteses como norteadoras das investigações e dos corpos de conhecimento, e dessa maneira, pode contribuir para uma visão deformada do trabalho científico por parte dos alunos."

Nesse sentido, o licenciando, em geral, vive uma situação dicotômica: por um lado em que lê e discute artigos afirmando que abordagens investigativas promovem melhora na aprendizagem, como auxiliar na construção de conceitos, ${ }^{10}$ no ensino de técnicas e procedimentos de laboratório, ${ }^{11}$ melhorar o raciocínio e habilidade de observação $;{ }^{12}$ por outro lado, quando tem as disciplinas experimentais do próprio curso, de maneira geral, vivenciam os experimentos com roteiros prontos. Assim, muitas vezes os objetivos, teorias e até mesmo as conclusões já estão explícitos, sendo que a justificativa para executar o experimento é a comprovação da teoria já estudada, o que vai de encontro com os objetivos de intenção de uma aula experimental.

Nesse contexto, o presente trabalho tem como objetivo apresentar um experimento com a proposta de uma abordagem investigativa para discutir conceitos de química e apresentar a técnica da espectrometria de massas a alunos de graduação. Para tanto, os conceitos serão explorados através da análise de óleo de soja e adulteração em azeite de oliva.

\section{Proposta}

O objetivo da aula experimental não seria apenas introduzir conceitos sobre a $M S$ utilizando a análise de óleos, mas sim abordar dentro da $M S$ conceitos importantes de química, como cálculos utilizados no preparo de soluções, calibração de instrumentos volumétricos, conceitos de ácidos e bases, conceitos de oxidação-redução e saturação e instauração de cadeias carbônicas. Enfim, conceitos importantes de química, e que podem ser abordados utilizando a $M S$ como plano de fundo, adequando o experimento aos objetivos da aula.

Todos os experimentos foram aplicados a alunos de iniciação científica do curso de Engenharia de Alimentos, com a possibilidade de transformar em uma aula experimental que poderia ser aplicada em aulas de Química Geral para as engenharias e aulas de Química Geral e Química Analítica para as turmas de Licenciatura em Química. Como forma de inserir os acadêmicos na problemática em questão foi proposta a situação problema a seguir.

Os óleos vegetais são constituídos principalmente de estruturas lipídicas, como os triacilgliceróis (TAGs) e, em menores quantidades, por ácidos graxos livres (AGs), monoacilgliceróis (MAGs) e diagilgliceróis (DAGs), que são formados a partir de processos de degradação, além de apresentarem outros componentes em pequenas quantidades, que variam de acordo com os tipos de óleos, como os esteróis, tocoferóis, fosfolipídios..$^{13,14}$

O óleo de soja é um produto muito comum na cesta básica dos brasileiros, podendo ser utilizado como óleo de cozinha, produção de margarinas, gordura vegetal, maionese, sendo que sua maior vantagem em relação a outros óleos é seu baixo custo de produção e comercialização. ${ }^{15,16}$ Já o azeite de oliva, mesmo apresentando excelentes propriedades nutricionais, não é tão comumente utilizado, e isso se deve ao fato de este tipo de azeite ter um elevado custo. Seu elevado valor comercial ocorre devido a uma menor produção em relação a outros óleos vegetais comestíveis. ${ }^{17}$ Por ser um óleo de alto valor comercial, é comum sua adulteração com óleo de soja.

A principal diferença entre o azeite de oliva e o óleo de soja é a composição dos TAGs presentes em cada tipo de óleo. O azeite de oliva apresenta em sua maioria uma composição em ácidos graxos formada por ácido oleico (55 a 83\%), ácido linoleico (3,5-21,0\%), palmítico (7,5-20\%), esteárico (0,5-5\%) e palmitoleico $(0,3-3,5 \%),{ }^{14}$ enquanto que no óleo de soja os TAGs em maior abundancia são formados pelos AGs: ácido linoleico $(49,7-56,9 \%)$, ácido oleico (17,7-26,0\%), ácido palmítico (9,9-12,2\%), ácido linolênico (5,5 $-9,5 \%)$ e ácido esteárico (3,0-5,4\%). A diferença no grau de saturação dos ácidos graxos que constituem o TAG é o que vai caracterizar a qualidade deste óleo, e a composição dos TAGs é que vai diferenciar as massas e qualificar cada um desses óleos. ${ }^{17}$

Existem diversas marcas no mercado que apresentam faixas de preço bastante distintas, causando duvidas no consumidor, se realmente a qualidade do produto é proporcional ao preço do mesmo. Considerando que os principais componentes dos óleos em AGs são o ácido linoleico (óleo de soja) e o ácido oleico (azeite de oliva), então surgem os questionamentos. Seria possível determinar a abundância de cada um desses compostos presentes nas amostras comerciais? Esses compostos podem ser utilizados como um indicativo que pode estar relacionado com a qualidade do produto?

A partir da problemática, sugere-se que os alunos levantem hipóteses de como seria possível fazer tal determinação. Com base nas hipóteses apresentadas, incentiva-se que sejam discutidos alguns critérios de diferenciação entre os produtos citados (óleo de soja e azeite de oliva) indicando a possibilidade de diferenciá-los por meio da identificação do principal componente de cada um deles, linoleico para o óleo de soja e o oleico para o azeite de oliva. Menciona-se, então, que existe uma técnica que é capaz de diferenciar tais substâncias por meio de suas massas moleculares, a espectrometria de massas.

Na sequência devem ser apresentados aos acadêmicos aspectos básicos da espectrometria de massas e também alguns espectros de massas simples. Deve-se então discutir como fazer a leitura desses espectros, bem como o significado físico dos "sinais" presentes no espectro de massas. Deve-se também apresentar as partes do equipamento, e dar ênfase à fonte de ionização utilizada, por se tratar de uma importante parte no espectrômetro de massas.

Uma das fontes de ionização mais utilizadas é a Ionização por electrospray (ESI). ${ }^{18-20}$ Nesse tipo de fonte de ionização, a função do electospray é criar pequenas gotas do solvente contendo as moléculas (algumas ionizadas outras não), em um processo chamado de nebulização. Após a nebulização da amostra, o solvente é removido pelo gás de dessolvatação, que age na superfície das gotas, evaporando o solvente e tornando as gotas menores, os íons formados que estão na superfície das pequenas gotas "saltam" para fase gasosa (mecanismo da evaporação de ions), ou então através de clivagens sucessivas das pequenas gotas chegam à fase gasosa (mecanismo do resíduo carregado). Finalmente, os íons são conduzidos para o analisador de massas (que mede as massas) através de diferenças 
de potencial atrativo e os mesmos, depois da análise, são detectados suas abundâncias são medidas. ${ }^{21}$

Na fonte de ESI podem ser gerados íons moleculares, moléculas protonadas/desprotonadas e moléculas cationizadas ou anionizadas. Para que se entenda a forma predominante, devem-se levar em conta os processos ocorridos no interior do capilar: reações de oxidação-redução produzem íons moleculares $\left(\mathrm{M}^{+}, \mathrm{M}^{+}\right)$ou $\left(\mathrm{M}^{-}, \mathrm{M}^{-}\right)$; já reações ácido/base resultam na formação de moléculas protonadas $[\mathrm{M}+\mathrm{H}]^{+}$ ou desprotonadas $[\mathrm{M}-\mathrm{H}]^{-}$. E os chamados adutos de Sódio e Potássio, que são devido à coordenação das moléculas do analito com cátions (geralmente os da família 1A) que leva à formação de moléculas cationizadas $\left([\mathrm{M}+\mathrm{Na}]^{+},[\mathrm{M}+\mathrm{K}]^{+}\right.$, ou ânions (principalmente cloretos), que leva à formação de moléculas anionizadas $\left.[\mathrm{M}+\mathrm{Cl}]^{-}\right){ }^{22}$

Após esta etapa, iniciou-se a parte experimental, na qual foram analisadas algumas amostras de óleos e azeites: 1 amostra de óleo de soja, 2 amostras de azeite de oliva, sendo uma de maior valor comercial e outra de menor valor, além de amostras adulteradas de azeite de oliva com óleo de soja, preparados no laboratório, com o objetivo de encontrar um perfil de adulteração.

\section{MATERIAIS E MÉTODOS}

\section{Materiais}

Foram utilizadas vidrarias comuns a laboratórios de química, como balão volumétrico, béqueres, provetas e micropipeta volumétrica. Para a solubilização das amostras utilizou-se um ultrassom (Ultracleaner 1400, Indaiatuba, Brasil) e um agitador de tubos tipo vortex (Fisaton, Perdizes, Brasil).

Para as análises foram utilizados um espectrômetro de massas Quatro Premier XE triplo quadrupolo (Waters Corporation, Milford, MA, USA) e o software MassLynx (Waters Corporation, Milford, MA, USA). As análises foram realizadas em modo de análise de íons positivos, com injeção de $5 \mu \mathrm{L}$ de amostra, na vazão de 0,20

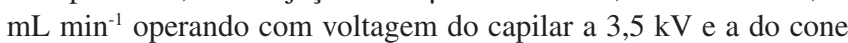
a $50 \mathrm{~V}$. A temperatura da fonte e de dessolvatação a $110{ }^{\circ} \mathrm{C}$ e a 400 ${ }^{\circ} \mathrm{C}$, respectivamente, fluxo do gás do cone $0 \mathrm{~L} \mathrm{~h}^{-1} \mathrm{e}$ fluxo do gás de dessolvatação $300 \mathrm{~L} \mathrm{~h}^{-1}$.

Metanol obtido da Vetec Sigma-Aldrich (Duque de Caxias, RJ), ácido fórmico, do inglês, formic acid $(F A)$, ácido trifluoroacético, do inglês, trifluoracetic acid (TFA) e éter 18-coroa-6 obtidos da Merck (Darmstadt, Germany). A água utilizada nos experimentos foi purificada utilizando sistema Milli-Q da Millipore (Bedford, MA, USA).
As amostras de óleo e de azeite de oliva foram compradas nos mercados locais. As amostras de azeite de oliva adulteradas com óleo de soja foram produzidas no laboratório na proporção de 50:50 (v/v) azeite de oliva:óleo de soja.

\section{MÉTODOS}

\section{Solução estoque de 18-coroa-6}

A solução foi preparada pesando $0,0339 \mathrm{~g}$ do éter 18-coroa-6 e solubilizando-o em $50 \mathrm{~mL}$ de metanol, com concentração final do éter de $0,68 \mathrm{mg} \mathrm{mL}^{-1}$. A solução foi estocada protegida da luz em temperatura de $0{ }^{\circ} \mathrm{C}$ para posterior utilização.

\section{Solução transportadora}

Em um balão de $100 \mathrm{~mL}$ adicionou-se $5 \mathrm{~mL}$ da solução estoque do éter 18-coroa-6 e completou-se o volume com metanol preparado com TFA na concentração de $0,1 \%$ (v/v), chegando na concentração final de éter 18 -coroa-6 $0,034 \mathrm{mg} \mathrm{mL}^{-1}$. Solução preparada para análise em modo positivo (DI-ESI(+)-MS).

\section{Solução estoque de óleo}

25,0 $\mu \mathrm{L}$ de uma amostra de óleo foram homogeneizados juntamente com $10 \mathrm{~mL}$ de metanol em um balão volumétrico e deixados em banho de ultrassom por 20 min para a solubilização dos triacilgliceróis.

\section{Preparo das soluções para análise}

A solução a ser injetada foi preparada em um tubo tipo eppendorf de $2 \mathrm{~mL}$, utilizando $50,0 \mu \mathrm{L}$ da solução estoque de óleo ou de azeite em 1950,0 $\mu \mathrm{L}$ da solução transportadora.

\section{RESULTADOS E DISCUSSÕES}

Para que os alunos pudessem compreender as análises, escolheu-se um sinal, referente a um TAG presente no óleo de soja para identificação deste óleo (triacilglicerol composto por 3 ácidos graxos linoleicos, $m / z$ 879) Figura 1, e um TAG específico para identificação do azeite de oliva (triacilglicerol composto por 3 ácidos graxos oleicos, $m / z$ 885) Figura 2.

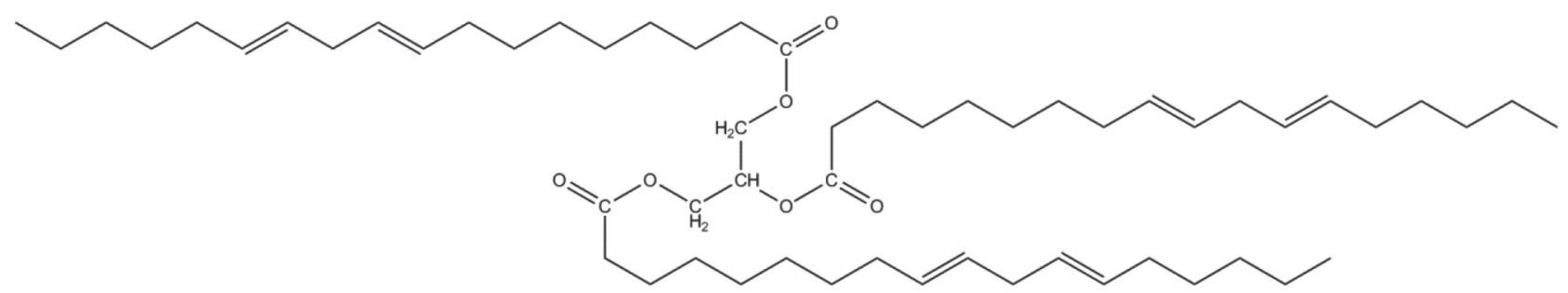

Figura 1. Estrutura do triacilglicerol característico de $\mathrm{m} / z$ 879 - LLL (L=Linoleico), o qual aparece com mais abundância no óleo de soja

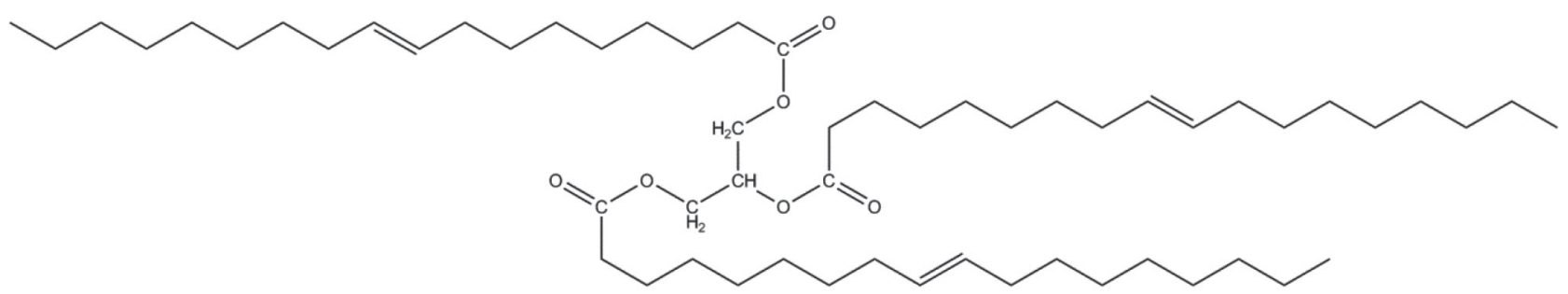

Figura 2. Estrutura do triacilglicerol característico de $\mathrm{m} / z, 885$ - OOO $(O=O$ Oleico $)$ o qual aparece com mais abundância no óleo de oliva 
O primeiro experimento apresentado aos alunos teve como objetivo mostrar a necessidade de acidificar a amostra quando se trabalha no modo positivo por ESI, para que a molécula a ser analisada seja protonada, obtendo assim um cátion $[\mathrm{M}+\mathrm{H}]^{+}$. A força dos ácidos influencia no mecanismo de protonação das moléculas, para que isto ficasse evidenciado, foram preparadas e injetadas duas amostras de óleo de soja: uma preparada em meio $F A$ (ácido mais fraco) e a outra amostra preparada em meio TAF (ácido mais forte) como mostrado na Figura $3 \mathrm{a}$ e 3b. Na presença de um ácido mais fraco, Figura 3a, o TAG de interesse (LLL) tem seu sinal distribuído entre moléculas protonadas $[\mathrm{M}+\mathrm{H}]^{+}\left(\mathrm{m} / \mathrm{z}\right.$ 879), sodiadas $[\mathrm{M}+\mathrm{Na}]^{+}(\mathrm{m} / \mathrm{z}$, 901) e potassiadas $[\mathrm{M}+\mathrm{K}]^{+}(\mathrm{m} / z$, 917). Na presença do ácido mais forte, Figura $3 \mathrm{~b}$, observou-se que os íons com $\mathrm{m} / \mathrm{z}, 879$ tiveram um aumento do sinal em relação aos íons de $\mathrm{m} / z 901$ e $\mathrm{m} / z, 917$, mostrando que a protonação com ácido mais forte é também mais eficiente.

Mesmo com o ácido mais forte sendo mais eficiente, observa-se no espectro de massas a presença de adutos de $\mathrm{Na}^{+} \mathrm{m} / z$, 901 (LLL) dos íons $[\mathrm{M}+\mathrm{Na}]^{+}$e adutos de $\mathrm{K}^{+}$de $\mathrm{m} / z 917$ (LLL) dos íons $[\mathrm{M}+\mathrm{K}]^{+}$, que fazem com que o sinal referente de $m / z, 879$ (LLL) dos íons
$[\mathrm{M}+\mathrm{H}]^{+}$se torne menor. Para diminuir ainda mais a divisão de íons entre espécies protonadas, sodiadas e potassiadas, adicionou-se o éter 18-coroa-6, que por ser um agente quelante, se coordena aos metais $\left(\mathrm{Na}^{+}\right.$e $\left.\mathrm{K}^{+}\right)$fazendo com que os sinais referentes a $[\mathrm{M}+\mathrm{Na}]^{+}$ $\mathrm{e}[\mathrm{M}+\mathrm{K}]^{+}$diminuam consideravelmente, com consequente aumento do sinal do analito de $m / z 879[\mathrm{M}+\mathrm{H}]^{+}$, como mostra a Figura 3c. Esses resultados indicam que o uso do éter melhora a análise através da coordenação com os íons metálicos presentes na solução. Com isso a molécula protonada será a única forma da molécula no modo positivo, melhorando o sinal analítico. ${ }^{23}$

$\mathrm{Na}$ Figura 4 estão apresentados espectros de massas das amostras de a) azeite de oliva de marca conceituada e b) óleo de soja, em que se avaliou a faixa de $\mathrm{m} / \mathrm{z}, 830$ a $\mathrm{m} / \mathrm{z}, 930$, faixa escolhida de acordo com os íons de TAGs, sendo que os principais TAGs avaliados na diferenciação desses óleos são os com $\mathrm{m} / \mathrm{z}, 885$ - OOO (O-Oleico), o qual aparece com mais abundância no azeite de oliva, e o TAG com $m / z 879$ - LLL (L-Linolêico), que apresenta-se em maior concentração no óleo de soja. As estruturas dos ácidos graxos são apresentadas nas Figuras 1 e 2, respectivamente.

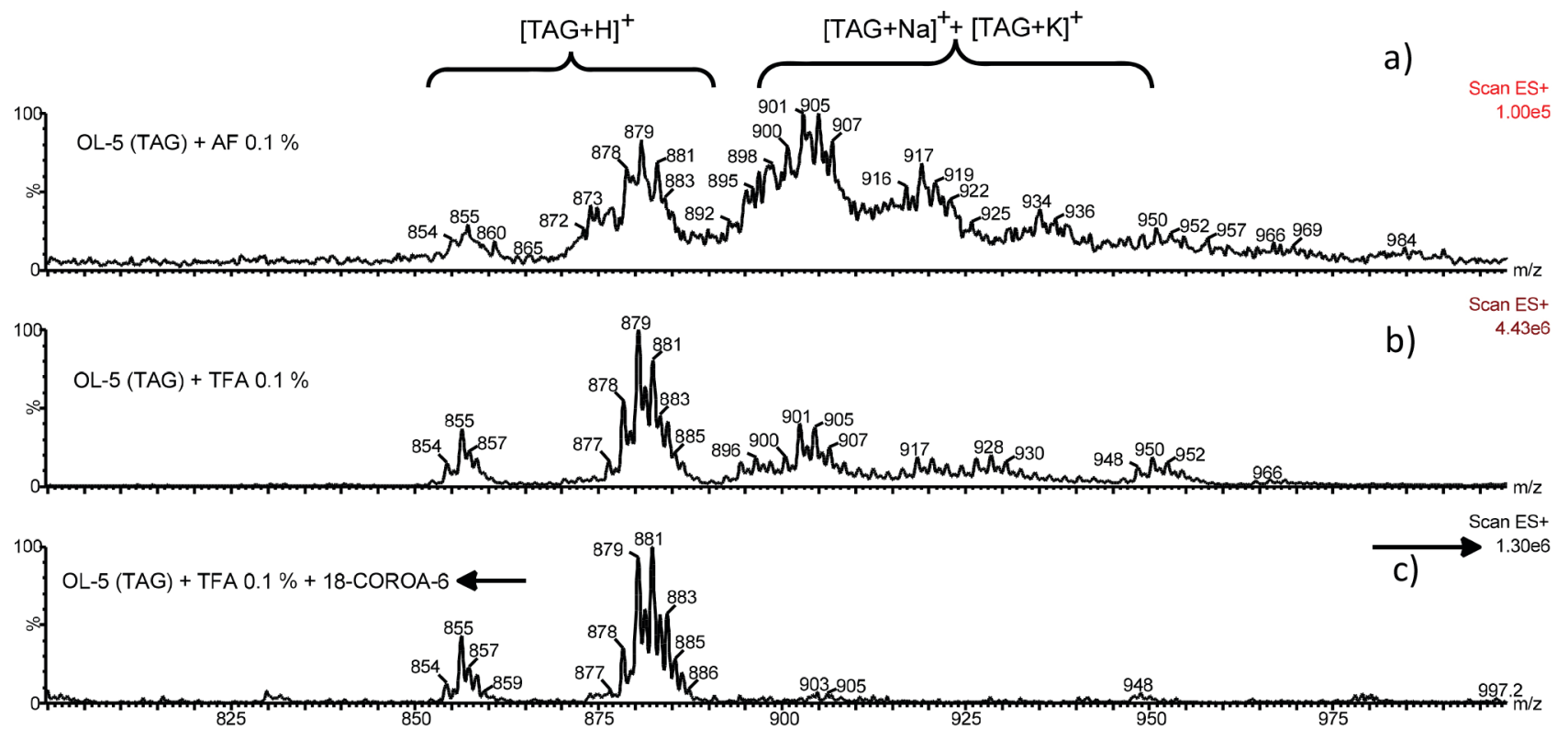

Figura 3. Espectros resultantes dos testes com Óleo de soja em a) Ácido Fórmico 0,1\% em metanol, b) Ácido Trifluoracético 0,1\% em metanol c) Ácido Trifluoracético 0,1\% e Éter18-coroa-6 (2,6 mmol/l ) em metanol

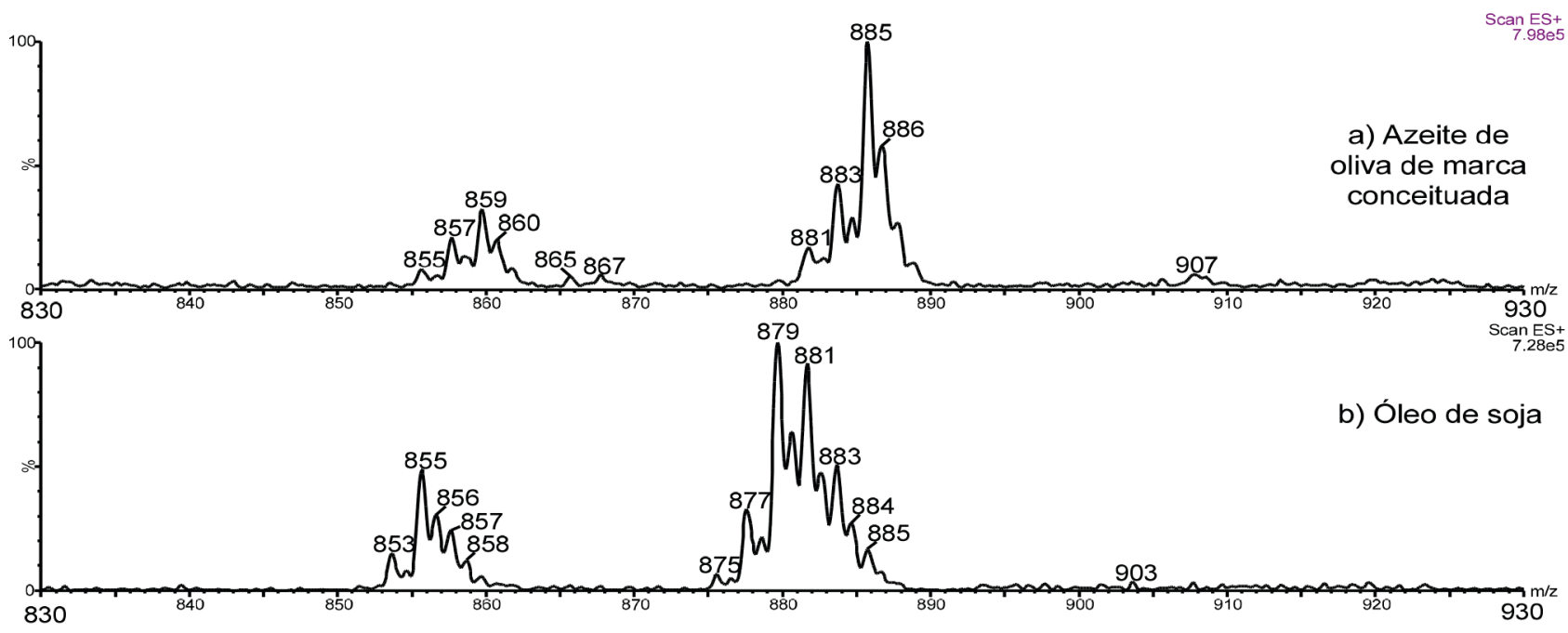

Figura 4. Espectros de massas do azeite de oliva de marca conceituada (a) e óleo de soja (b) 

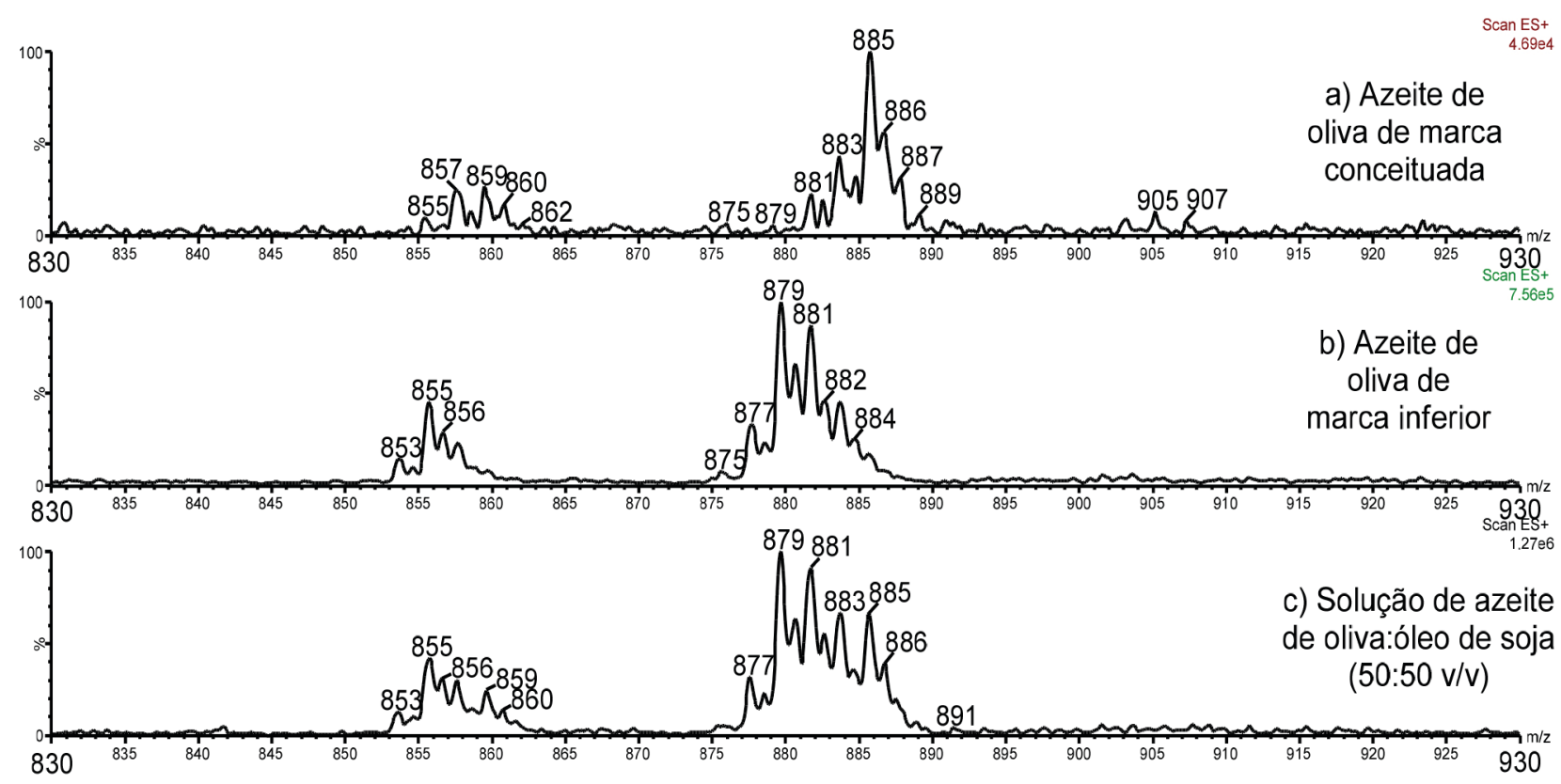

Figura 5. Espectros de massas azeite de oliva de marca conceituada (a), azeite de oliva de marca inferior (b) e solução azeite de oliva: óleo de soja 50:50 v/v (c)

Na Figura 4, podemos identificar as similaridades e diferenças entre os dois tipos de óleos vegetais analisados, sendo as diferenças representadas pelos íons com maior abundância $m / z, 885$ para o azeite de oliva e $m / z, 879$ para o óleo de soja. Essas duas massas podem ser marcadores de presença de cada óleo. Esses marcadores podem ser utilizados para identificar adulteração em azeite de oliva.

A Figura 5 mostra os espectros de massas de dois azeites de oliva comprados em um mercado local, a) de marca reconhecida e b) de marca inferior, além de c) solução azeite de oliva: óleo de soja (50:50 v/v). Quando comparamos os espectros do azeite de oliva de marca inferior 5 b) com o espectro do óleo de soja puro 4 b) e a solução azeite de oliva: óleo de soja (50:50 v/v) 5 c), observamos uma grande similaridade nos sinais obtidos, sendo o sinal mais abundante apresenta o íon $[\mathrm{M}+\mathrm{H}]^{+}$de $\mathrm{m} / \mathrm{z} 879$ que é característico de óleo de soja, isso nos leva a certeza de que este azeite de oliva de marca inferior apresenta grande quantidade de TAG-(LLL) e que ele pode sim ter sido adulterado com óleo de soja. Podemos ainda inferir que o azeite de oliva de marca inferior deve apresentar mais que 50\% de óleo de soja, já que o cation de $m / z, 885$ tem uma abundância menor que o azeite de oliva adulterado em laboratório (Figura 5 c)) e próxima a abundância do óleo de soja puro (Figura 4b)).

Com a análise dos espectros, os acadêmicos passam a ter contato com os resultados apresentados e, diferente da forma como geralmente se abordam os conceitos referentes à espectrometria de massas, utilizam os dados para resolver uma situação problema. Dessa forma os acadêmicos precisam interpretar os espectros e só assim conseguirão solucionar o problema proposto. Assim, a compreensão dos conceitos inerentes à espectrometria de massas (objetivo da disciplina) por parte dos estudantes possivelmente ocorrerá de forma mais efetiva e natural, deixando de enfatizar os aspectos de memorização sobre o aparelho e suas partes e focando na aplicação e nos resultados.

Com relação a outros sinais observados no espetro dos óleos e azeites, pode-se oferecer ao aluno a Tabela 1, com massas de alguns TAGs, para que ele tente verificar quais os possíveis íons encontrados nas amostras.

\section{Valor educacional}

Um ganho com o uso de uma abordagem problematizadora,
Tabela 1. Principais TAGs e adutos verificados pelo experimento proposto atribuídos através da utilização da plataforma LipidMaps ${ }^{\mathrm{R}}$. Onde os principais ácidos graxos que compõem os TAGs são: $\mathrm{Po}=$ Palmitoleico, $\mathrm{O}=$ oleico, L=linoleico, $\mathrm{P}=$ Palmítico, $\mathrm{Ln}=$ Linolênico, $\mathrm{S}=$ esteárico

\begin{tabular}{lccc}
\hline TAG & {$[\mathrm{M}+\mathrm{H}]^{+}$} & {$[\mathrm{M}+\mathrm{Na}]^{+}$} & {$[\mathrm{M}+\mathrm{K}]^{+}$} \\
\hline PoLL & 853 & 875 & 891 \\
PLL & 855 & 877 & 893 \\
POL & 857 & 879 & 895 \\
POO & 859 & 881 & 897 \\
LLLn ou OLnLn* & 877 & 899 & 915 \\
LLL ou OLLn* & 879 & 901 & 917 \\
OLL ou OOLn* & 881 & 903 & 919 \\
SLL & 883 & 905 & 921 \\
OOO & 885 & 907 & 923 \\
OOS & 887 & 909 & 925
\end{tabular}

Nota: Os íons com asteriscos são os de menores abundancia nos óleos. ${ }^{17}$

utilizando a análise de azeite de oliva possivelmente adulterado com óleo de soja por espectrometria de massas, são os conceitos básicos de química que podem ser abordados durante toda a aula ou após os experimentos. É importante que se fale dos cálculos necessários para o preparo de soluções, padrões e das amostras, a calibração dos instrumentos como balões e pipetas volumétricas, conceitos básicos para a experimentação em aulas de química.

Como as análises foram feitas todas no modo positivo, foram testados dois ácidos: FA e TFA. Aproveitando-se deste tema, pode-se trabalhar com os alunos conceitos de ácido-base, reações em meio aquoso e constante de equilíbrio. E através das massas obtidas, pode-se observar de forma clara os prótons que foram adicionados às moléculas através das reações ácido base.

Outro conceito que se pode trabalhar com os alunos é sobre saturação e instauração de cadeias. Os óleos analisados apresentam constituição dos TAGs principais com cadeias carbônicas distintas, e por isso podem ser identificados, e isso também pode ser utilizado em sala de aula enriquecendo ainda mais o conteúdo abordado com 
o experimento. Assim como o conceito de massa molecular, visto que se trata de um assunto que muitas vezes confunde os alunos e pode ser desmistificado analisando os espectros de massas. Enfim, com a finalidade de analisar possíveis adulterações em óleo de oliva, consegue-se inserir uma grande quantidade de assuntos, sendo que o principal são as análises por espectrometria de massas, porém, o experimento não é o fim, e sim o início de uma construção/consolidação de conhecimentos que podem ser adquiridos durante uma aula.

\section{CONSIDERAÇÕES FINAIS}

Com essa atividade experimental diferenciada espera-se que os acadêmicos que cursam disciplinas de química em cursos de engenharias e, em especial, os licenciandos em Química compreendam os conceitos básicos inerentes à espectrometria de massas, e vislumbrem o amplo espectro de aplicações que essa técnica apresenta. Além disso, a abordagem apresenta um viés investigativo, trazendo uma problematização seguida pelo levantamento de hipóteses dos alunos para que, enfim, seja apresentada a técnica abordada como uma forma de resolver o problema proposto. Dessa forma, o acadêmico precisa avaliar os dados obtidos para concluir se pode ou não diferenciar as amostras e como isso deve ser feito.

Para os alunos que cursam a Licenciatura, esse tipo de abordagem se torna ainda mais relevante, pois ele vivencia, enquanto aluno, uma tendência de ensino que se discute nas disciplinas específicas, porém em geral, não se aplica em situações reais de ensino. Para o professor, trata-se de um experimento simples (apenas 6 injeções, no máximo 30 min destinados ao preparo da amostra e 1,0 min por injeção) que pode ser aplicado em duas aulas experimentais.

\section{REFERÊNCIAS}

1. Dass, C.; Fundamentals of contemporary mass spectrometry, John Wiley \& Sons: New Jersey, 2007.

2. Kim, D. H.; Eckhert, C. D.; Faull, K. F.; J. Chem. Educ. 2011, 88, 106.

3. Harmon, C. W.; Mang, S. A.; Greaves, J.; Finlaysin-Pitts, B.; J. Chem. Educ. 2010, 87, 186.

4. Beglinger, K. M.; Wormwood, K. L.; Mattingly, J. W.; Larlee, C. R.; Wawro, B. M.; Woods, B.; Darie, C. C.; Mod. Chem. Appl. 2013, 112, 1.
5. Golge, O.; Kabak, B.; Food Chem. 2015, 176, 319.

6. Afify, A. E.-M. M. R.; Mohamed, M. A.; El-Gammal, H. A.; Attallah, E. R.; J. Food, Agric. Environ. 2010, 8, 602.

7. Simas, R. C.; Tese de doutorado, Universidade Estadual de Campinas, Brasil, 2010.

8. Sá, E. F.; Quadros, A. L.; Mortimer, E. F.; Silva, P. S.; Talim, S. L.; Rev. Bras. Educ. 2017, 22, 625.

9. Suart, R.; Marcondes, M. E. R.; VI Encontro Nacional de Pesquisa em Educação em Ciências, Florianópolis, Brasil, 2007.

10. Ferreira, L. H.; Hartwig, D. R.; Oliveira, R. C.; Quim. Nova Esc. 2010, 32, 101.

11. Borges A. T.; Cad. Brás. Ens. Fís. 2002, 9, 291.

12. Galiazzi, M. C.; Rocha, J. M. B.; Schmitz, L. C.; Souza, M. L.; Giesta, S.; Gonçalves, F. P.; Ciênc Educ. 2001, 7, 249.

13. Wu, Z.; Rodegers, R. P.; Marshall, A. G.; J. Agric. Food Chem. 2004, 52, 5322; Eldin, A. K.; Eur. J. Lipid Sci. Technol. 2006, 58, 1051.

14. Gunstone, F. G.; Vegetable Oils in food technology composition, properties and use, $2^{\text {nd }}$ ed., Wiley-Blackwell: New Jersey, 2011, cap. 3 and 9.

15. Justino, A. L.; Lage, M. M. R.; Pereira, T. G. G.; Rodrigues, M. F.; Silva, M. A.; Orlandi, D.; Maia, G.; Sales, A.; Queiroz, B.; Oliveira, J.; Cabral, M.; Epifanio, Y.; Walter, M. E.; Abi-Saber, Ã.; Exacta 2011, 4, 19.

16. Peixoto, E. R. M.; Santana, D. M. N.; Abrantes, S.; Ciênc. Tecnol. Aliment. 1998, 18, 444

17. Simas, R. C.; Catharino, R. R.; Cunha, I. B. S.; Cabral, E. C.; BarreraArellano, D.; Eberlin, M.N.; Albereci, R. M.; Analyst 2010, 135, 738.

18. Aquino, F. J. T.; Augusti, R.; Alves, J. O.; Diniz, M. E. R.; Morais, S. A. L.; Alves, B. H. P.; Nascimento, E. A.; Sabino, A.; Microchem. J. 2014, 117,127

19. Gross, D. S.; Ryswyk, H. V.; J. Chem. Educ., 2014, 91, 1240.

20. Fenn, J. B.; Mann, M.; Meng, C. K.; Wong, S. F.; Whitrhouse, C. M.; Science 1989, 246, 64.

21. Gross, J. H.; Mass Spectrometry, $1^{\text {st }}$ ed., Springer: Heidelberg, 2004, cap. 11.

22. Crotti, A. E. M.; Vessecchi, R.; Lopes, J. L. C.; Lopes, N. P.; Quim. Nova 2006, 29, 287.

23. Tonin, A. P. P.; Ribeiro, M.; Silva, V. M.; Oliveira, C. C.; Saragiotto, M. H.; Eberlin, M. N.; Meurer, E. C.; Int. J. Mass Spectrom., 2017, 418, 37. 